\title{
Adsorption of associating fluids at active surfaces: a density functional theory
}

\author{
S.Tripathi, W.G.Chapman* \\ Department of Chemical Engineering, Rice University, \\ Houston, TX 77005, USA
}

Received May 7, 2003, in final form August 4, 2003

\begin{abstract}
We present a density functional theory (DFT) to describe adsorption in systems where molecules of associating fluids can bond (or associate) with discrete, localized functional groups attached to the surfaces, in addition to other fluid molecules. For such systems as water adsorbing on activated carbon, silica, clay minerals etc. this is a realistic model to account for surface heterogeneity rather than using a continuous smeared surface-fluid potential employed in most of the theoretical works on adsorption on heterogeneous surfaces. Association is modelled within the framework of first order thermodynamic perturbation theory (TPT1). The new theory accurately predicts the distribution of bonded and non-bonded species and adsorption behavior under various conditions of bulk pressure, surface-fluid and fluid-fluid association strengths. Competition between the surface-fluid and fluid-fluid association is analyzed for fluids with multiple association sites and its impact on adsorption is discussed. The theory, supported by simulations demonstrates that the extent and the nature of adsorption (e.g. monolayer) vary with the number of association sites on the fluid molecules.
\end{abstract}

Key words: adsorption, associating fluids, active surfaces, density functional theory

PACS: 68.35.Dv, 68.43.-h, 68.43.De, 68.43.Fg, 71.15.Mb

\section{Introduction}

Most of the theoretical studies describing interactions of complex fluids with solid surfaces have been restricted to models of homogeneous pores and surfaces, which means that the external field was a function only of the distance normal to the surface. While these idealistic models provide valuable insights into the general phase behavior of the system, they nevertheless fall short of allowing a direct comparison

*Author for correspondence - e-mail: wgchap@rice.edu, phone: 1-713-348-4900, fax: 1-713$348-5478$ 
with experiments where the real microporous surfaces are marked with heterogeneity (in geometry and/or chemistry) due to the presence of impurities, dislocation and imperfections. Geometric heterogeneity results from non-uniformity in the pore shape or width, network effects or finite-size effects while chemical or energetic heterogeneity results from the variation in the adsorption potential across the surface. In this work, we focus on energetic heterogeneity, which is of wide practical interest (selective adsorption, separations, catalysis).

Energetic surface heterogeneity can itself be of two types, (a) continuous and (b) discrete. In the type (a), the adsorbing potential is smeared-out or continuous (at least over a certain finite region) across the surface. This can be as simple as the surface being divided into strips or grids or other regular well-defined patterns of weakly or strongly adsorbing patches. In other words, characterized by continuously varying unidirectional adsorbing fields. This type of heterogeneity has been studied in reasonable detail through both simulations and theories. Chmiel et al. [1] have used the Tarazona density functional theory (DFT) $[2,3]$ to study a Lennard-Jones fluid against a wall formed of parallel strips possessing different adsorbing energies. Using continuous and lattice DFT, Rocken et al. $[4,5]$ have shown that the phase behavior of the LJ fluid is significantly influenced due to periodic energetic corrugation. Huerta et al. [6] and Zygmunt et al. [7] have applied the 3D form of the associating fluid DFT of Segura et al. [8-10] to describe the phase behavior of associating fluids in energetically heterogeneous pores with periodic adsorbing potential.

Discrete heterogeneity, on the other hand, occurs where the surface is doped with certain active sites or functional groups that can bond or associate with the fluid molecules resulting in a localized discontinuity in the surface fluid interaction. Graphite pores functionalized with hydroxyl (OH-) or carboxyl (COO-) groups are typical examples. The adsorption on these surfaces is strongly affected by the presence, type and density of active sites on the solid surfaces to which the adsorbate fluid molecules can associate. A fundamental understanding at molecular level of such systems would extremely enhance our ability to modify and control the adsorption of associating molecules through appropriate doping of the surfaces.

Systems exhibiting this form of surface heterogeneity have been primarily studied through molecular simulations. Muller, Vega and coworkers [11-14] have reported a Monte Carlo study of water adsorption on activated carbons, where water molecules have been modelled as Lennard-Jones (LJ) spheres with four square-well sites. They have shown that the effect of wall fluid association can strongly influence the adsorption characteristics of water, as much as changing the slit pore from desorption to adsorption in certain cases. McCallum et al. [15] also used a similar water model and molecular simulation to study the adsorption isotherm of water onto activated carbons at low pressures. Muller et al. [14] presented the adsorption isotherms of associating LJ chain molecules against an LJ surface with active sites using Monte Carlo simulations. This study gave some interesting results for the consequence of competition between the tendencies of the individual spherical segments of a chain to place themselves in the center of the pore versus the strongly favorable bonding position near the wall. Lee and Rossky [16] investigated, using molecular dynam- 
ics simulations, the structure and dynamics of liquid water at three types of solid surfaces: flat hydrophobic, rough hydrophobic, and hydrophilic. They found that the two hydrophobic surfaces exhibited similar behavior. For the hydrophilic wall, surface activity was stronger than the bulk interaction between the molecules. In all cases, structural perturbations are greatly diminished at distances greater than 2 or 3 molecular layers from the wall. Segura and Chapman [8] presented a comparison between the structure of a water-like fluid near hydrophobic and hydrophilic surfaces.

This form of surface heterogeneity has also been studied to some extent using integral equation (IE) theories. Kovalenko et al. [17] have used Percus-Yevick (PY1) / associative Percus-Yevick (APY) approximation to singlet level HendersonAbraham-Barker (HAB) equations to calculate density profiles of associating fluids in crystalline slit-pores with bonding sites. Vakarin et al. [18] have used a similar approach to study the role of molecular aggregation and determine the structural and connectivity properties of a network forming fluid near a crystalline surface. Surface-fluid association in their work has been modelled as a sticky lattice potential, hence ignoring the orientation dependence of association. Issues related to phase transitions are difficult to address within the IE framework, since the singlet level theories are incapable of describing phase transitions and pair level theories are difficult to implement, more so for crystalline surfaces [17]. Moreover the problem of finding accurate closures has always been a serious limitation of the IE based studies of inhomogeneous systems. Non-local DFTs have gained significant interest in recent times due to their superior accuracy, relatively simpler form and better thermodynamic consistency compared to IE theories. Clay minerals such as kaolinite allow the study of two chemically different surfaces, one covered with hydroxyl groups and the other one covered with oxygen atoms. Adsorption (esp. of water) in these systems has been studied through molecular dynamics and Monte Carlo methods [19-21] and using quantum chemical calculations [22-24]. A fairly detailed review of theoretical work on adsorption in clay minerals is contained in [22] and the references therein.

We have developed a non-local DFT to account for the discrete form of surface heterogeneity using the ideas derived from the DFT of Segura et al. [9,10], combining Tarazona's DFT [2,3] for the excluded volume and Wertheim's TPT1 [25-28] for the association interactions. Our confidence in this prescription of Segura et al. stems from its numerous successful applications reported in the literature, as discussed in [29]. However, the coupling of the hard sphere DFT and TPT1 is done in such a way that the use of alternate forms of DFTs for the excluded volume such as Meister-Kroll [30] and Rosenfeld [31] should not pose any formal problem. The detailed derivation of our theory can be found elsewhere $[32,33]$. The theory is derived in two stages. In the first stage [32] it describes a system where the fluid molecules can associate only with surface sites and not among themselves. This limiting case allows us to isolate the effects of wall-fluid association. Study of these wall-fluidbonding systems as such is useful in understanding biochemical interactions near membranes, where molecules can bond only with the surface, as in biosensors. The inclusion of the contributions from fluid-fluid association in the next stage com- 
pletes the derivation [33]. Here we present the theory in its final derived form, for a hard sphere fluid with $M_{\text {sites }}$ association sites in a pore with hard walls having $N_{\mathrm{W}}$ active sites each. The hard sphere system was chosen to allow direct comparison with molecular simulations. We discuss some of the features of the theory and demonstrate its success in predicting the adsorption and averaged distribution of bonded and non-bonded species away from the surface, in the above system. While computing averaged properties is straightforward for smooth surfaces, systems with a heterogeneous surface system require a $2 \mathrm{D}$ or $3 \mathrm{D}$ solution of the DFT, where the complexity of the numerical problem increases dramatically, to the extent that it calls for the use of parallel algorithms. To quote the result of Frink and Sallinger $[34,35]$, the number of operations required for a Jacobian fill in a 2D problem was $O\left(10^{9}\right)$, compared to $O\left(10^{5}\right)$ for the corresponding $1 \mathrm{D}$ problem. Although the payoff of the added complexity is the 3D structure of the fluid near the surface, the computation of even the averaged properties such as adsorption and density distribution away from the wall, requires one to go through the rigorous solution of the 3D problem. Orientation dependence of association interactions (surface-fluid and fluid-fluid) further aggravates the problem. Our theory computes adsorption and averaged distribution of total, bonded and non-bonded species away from a discretely heterogeneous surface, without actually solving a 3D DFT formalism. In a separate paper [32], we have shown that in addition to the predictions for the plane-averaged properties, the theory can also provide a qualitative estimate of the 3D structure (or the in-plane structure) of the fluid, all at the computational expense of a 1D DFT.

We start with outlining in the next section, the potential model and a brief description of simulation methodology. Next we present the theory for a hard sphere fluid with $M_{\text {sites }}$ association sites against a hard wall with $N_{\mathrm{W}}$ active sites. The hard sphere system was chosen to allow direct comparison with molecular simulations. In the next section, results for total and monomer density distribution, surface coverage, adsorption are discussed and compared with Monte Carlo (MC) simulations, first for only fluid-surface association and subsequently in the presence of fluid-fluid association.

\section{Potential model}

The system comprises spherical fluid molecules, each with $M_{\text {sites }}$ association sites arranged symmetrically on a hard sphere core, confined between two parallel hard walls in the $x-y$ plane. The separation between the walls can be adjusted to study the system behavior near the solid-fluid interface or its phase behavior in a narrow pore. In this particular work this separation is kept large enough to ensure a bulk like region near the pore center, free from any surface effects. The walls are taken as smooth, continuum solid surfaces passing through the centers of the atoms forming the surface layers of the walls. These structureless surfaces are activated by placing association sites in a square lattice on the walls, as shown in figure 1. These surface sites have identical geometric and energetic parameters to the association sites on the fluid molecules. 


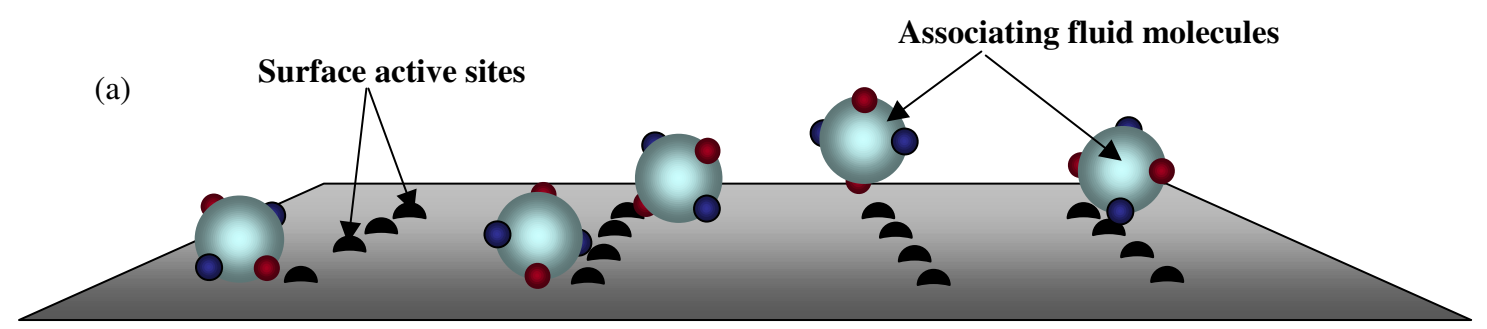

(b)

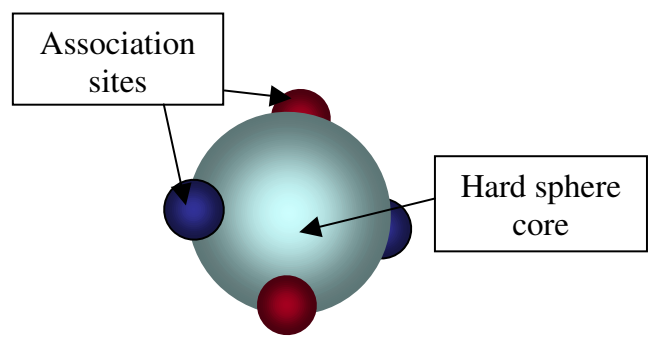

(c)

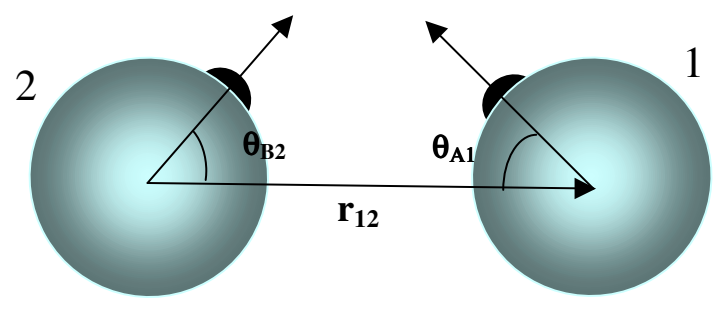

Fluid Fluid Association

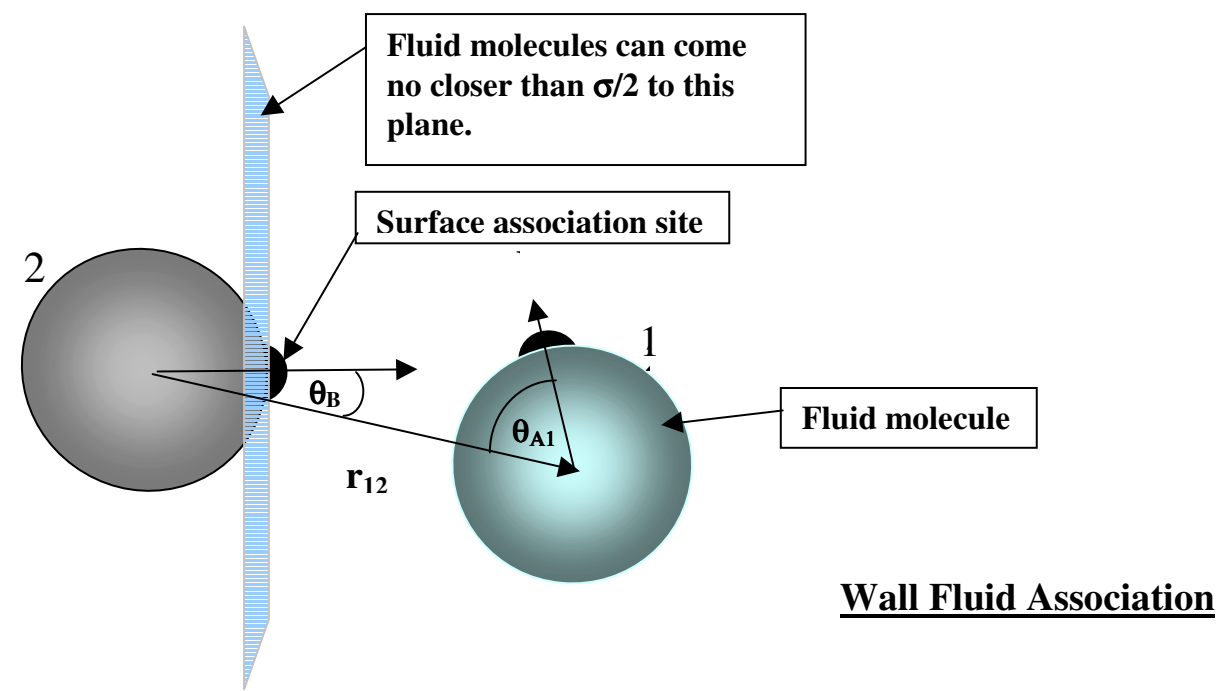

Figure 1. (a) Illustration of the surface with a distribution of association sites on a square lattice. (b) Schematic of a 4-site water-like associating fluid molecule showing tetrahedral arrangement of sites on a hard core. (c) Sketch showing the parameters required in the fluid-fluid and fluid-wall association potential model. 
The potential of interaction between two fluid molecules 1 and 2, is given by

$$
\phi_{12}^{\mathrm{FF}}\left(\mathbf{r}, \boldsymbol{\omega}_{1}, \boldsymbol{\omega}_{2}\right)=\phi_{12}^{\mathrm{R}}(r)+\sum_{A}^{M_{\text {sites }}} \sum_{B}^{M_{\text {sites }}} \phi_{A B}^{\mathrm{FF}, \text { assoc }}\left(\mathbf{r}, \boldsymbol{\omega}_{1}, \boldsymbol{\omega}_{2}\right),
$$

where $\phi_{12}^{\mathrm{R}}(r)$ is the pair potential of the reference fluid, which in our case is a hard sphere system. $\phi_{A B}^{\mathrm{FF}, \text { assoc }}\left(\mathbf{r}, \boldsymbol{\omega}_{1}, \boldsymbol{\omega}_{2}\right)$ is the contribution to the pair potential due to association between site $A$ on molecule 1 and site $B$ on molecule 2 and is given by the following square well potential

$$
\phi_{A B}^{\mathrm{FF}, \text { assoc }}\left(\mathbf{r}, \boldsymbol{\omega}_{1}, \boldsymbol{\omega}_{2}\right)=\left\{\begin{array}{cl}
-\varepsilon_{\mathrm{ff}}, & \text { if } \quad r<r_{C} ; \theta_{A 1}<\theta_{C}, \theta_{B 2}<\theta_{C}, \\
0, & \text { otherwise },
\end{array}\right.
$$

where $\sigma$ is the hard sphere diameter and $r$ is the magnitude of the vector $\mathbf{r}$ connecting the centers of molecules 1 and 2 , and $\boldsymbol{\omega}_{1}$ and $\boldsymbol{\omega}_{2}$ are the sets of angles defining the orientations of molecules 1 and 2 relative to vector $\mathbf{r}$. $\theta_{A 1}$ is the angle made by the vector from the center of molecule 1 to site $A$ and the vector $\mathbf{r}$ and $\theta_{B 2}$ is the angle between the vector from the center of molecule 2 to its association site $B$, and the vector $\mathbf{r}$, as shown in the figure 1 . The summation in equation (1) is over all possible combinations of association sites on the two molecules that can bond. $r_{C}$ and $\theta_{C}$ are the radial and angular limits of the square well interactions.

Fluid molecules interact with the active sites on the surfaces through the following potential

$$
\phi^{\mathrm{FW}}\left(\mathbf{r}_{1}\right)=\sum_{i}^{N_{\mathrm{W}}} \sum_{A}^{M_{\text {sites }}} \phi_{A}^{\mathrm{FW}, \text { assoc }}\left(\mathbf{r}_{i 1}, \boldsymbol{\omega}_{1}\right)
$$

$\phi_{A}^{\mathrm{FW} \text {,assoc }}\left(\mathbf{r}_{i 1}, \boldsymbol{\omega}_{1}\right)$ is the contribution due to association between site A on molecule 1 and the wall site $i$, given by the following square well potential

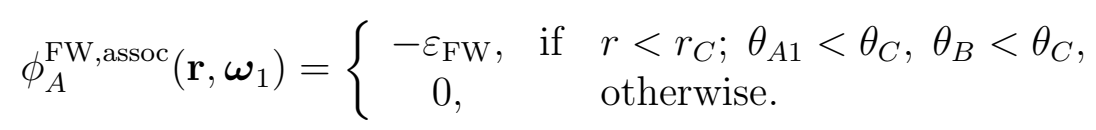

For the sake of argument, consider the wall site $i$ as being mounted on some surface molecule 2, having the same size as the fluid molecules, as shown in figure 1. $r$ is then the magnitude of the vector $\mathbf{r}$ connecting the centers of molecules 1 and 2 , and $\boldsymbol{\omega}_{1}$ is the set of angles defining the orientations of the fluid molecule 1 relative to vector $\mathbf{r}$. $\theta_{A 1}$ and $\theta_{B}$ are the angles made by the vectors from the centers of the molecules 1 and 2 to their respective association sites, and the vector $\mathbf{r}$, as shown in the figure 1c. $r_{C}$ and $\theta_{C}$ are the radial and angular limits of the square well interaction. The inner and outer summations in (3) run over all association sites on the fluid molecules and all the active sites on the wall, respectively.

In addition to the association interaction through the active sites, the walls also exert an external field. The walls being in the $x-y$ plane, $z$ is the axis normal to the wall and the external potential is given by

$$
\phi^{\mathrm{Ext}}(z)=\left\{\begin{array}{cl}
\infty & \text { if } \quad z \leqslant 0 \text { or } z \geqslant H \\
0 & \text { otherwise }
\end{array}\right.
$$


where $\mathrm{H}$ is the separation between the walls.

We have performed Monte Carlo (MC) simulations in the NVT ensemble to compare the predictions of the theory. The potential model described above is used in simulations as well. The details of the simulation and averaging scheme can be found in [8].

\section{The free energy functional}

The DFT of Segura et al. [9,10] accurately describes a system of associating hard spheres against smooth hard walls. The particular approach adopted in our theory ensures that in the limit of smooth walls (no wall fluid association) it reduces to the Segura DFT. The excess (over ideal gas) free energy of the fluid is written as the sum of a hard sphere and a perturbative association term

$$
F^{\mathrm{ex}}[\rho(\mathbf{r})]=F^{\mathrm{ex}, \mathrm{hs}}[\rho(\mathbf{r})]+F^{\mathrm{ex}, \text { assoc }}[\rho(\mathbf{r})]
$$

The hard sphere excess energy is accounted for in the weighted density approximation as

$$
F^{\mathrm{ex}, \mathrm{hs}}[\rho(z)]=\int \mathrm{d} z \rho(z) \psi^{\mathrm{hs}}[\bar{\rho}(z)]
$$

where $\bar{\rho}(z)$ is Tarazona's weighted density $[2,3] . \psi^{\mathrm{hs}}[\bar{\rho}(z)]$ is the excess over the ideal gas free energy per molecule of a non-associating hard sphere. The association contribution comes from Wertheim theory

$$
\begin{aligned}
F^{\mathrm{ex}, \text { assoc }}[\rho(z)] & =M_{\text {sites }} \int \mathrm{d} z \rho(z) \psi^{\mathrm{assoc}}[\rho(z)] \\
\psi^{\mathrm{assoc}}[\rho(z)] & =k T \sum_{A}\left[\ln \chi_{A}^{\mathrm{F}}(z)-\frac{\chi_{A}^{\mathrm{F}}(z)}{2}+\frac{1}{2}\right],
\end{aligned}
$$

where the summation is over all association sites on a molecule. $\chi_{A}^{\mathrm{F}}(z)$ is the fraction of fluid molecules at $z$, not bonded at site A given by

$$
\begin{aligned}
\chi_{A}^{\mathrm{F}}(z) & =\left[1+\Gamma(z) \chi^{\mathrm{W}}+\Phi(z) \int_{z-\sigma}^{z+\sigma} \chi_{A}^{\mathrm{F}}\left(z_{2}\right) \rho\left(z_{2}\right) \mathrm{d} z_{2}\right]^{-1}, \\
\Gamma(z) & =\frac{N_{\mathrm{W}}}{S_{\mathrm{W}}} \pi\left(r_{C}^{2}-z^{2}\right) g_{\mathrm{fw}}^{\mathrm{ref}}(z) \frac{\left(1-\cos \theta_{C}\right)}{2}\left[\exp \left(-\beta \varepsilon_{\mathrm{fw}}\right)-1\right] \\
\Phi(z) & =\pi\left(1-\cos \theta_{C}\right)^{2}\left(r_{C}-\sigma\right) \sigma^{2} g_{\mathrm{ff}}^{\mathrm{ref}}\left(\sigma, \rho_{b}\right)\left[\exp \left(-\beta \varepsilon_{\mathrm{ff}}\right)-1\right]
\end{aligned}
$$

where $N_{\mathrm{W}} / S_{\mathrm{W}}=\sigma^{2} / a^{2}$ is the 'wall site density', where $a$ is the spacing between sites for evenly distributed surface sites, representing the site-site separation on the active surface. $\varepsilon_{\mathrm{fw}}$ and $\varepsilon_{\mathrm{ff}}$ are the fluid-wall (F-W) and fluid-fluid (F-F) association strengths respectively. $g_{\mathrm{fw}}^{\mathrm{ref}}(z)$ is the value of the wall-fluid radial distribution function in the reference fluid (non-associating hard sphere) at a separation $z$ from the wall. $g_{\mathrm{ff}}^{\mathrm{ref}}\left(\sigma, \rho_{b}\right)$ is the contact value of reference fluid pair correlation function at bulk 
density $\rho_{b}$. The second and third terms in the denominator of equation (10) denote contributions from F-W and F-F association respectively. $\chi^{\mathrm{W}}$ is the fraction of nonbonded wall sites given by

$$
\begin{aligned}
\chi^{\mathrm{W}}=\{1+ & \pi \frac{\left(1-\cos \theta_{C}\right)}{2}\left[\exp \left(-\beta \varepsilon_{\mathrm{fw}}\right)-1\right] \\
& \left.\times \sum_{A} \int_{\sigma}^{r_{C}} \rho\left(z_{1}\right) \chi_{A}^{\mathrm{F}}\left(z_{1}\right)\left(r_{C}^{2}-z_{1}^{2}\right) g_{\mathrm{fw}}^{\mathrm{ref}}\left(z_{1}\right) \mathrm{d} z_{1}\right\}^{-1} .
\end{aligned}
$$

The integration in the above equation is over the bonding range of the wall sites.

The detailed derivation of expressions for $\chi_{A}^{\mathrm{F}}$ and $\chi^{\mathrm{W}}$ starting from Wertheim's theory, highlighting all the assumptions and simplifications can be found in [32]. Although equation (10) gives the average value of the fraction of non-bonded specie at any distance away from the wall, it is important to note that the non-uniformity in fluid properties in the planes parallel to the wall is not neglected. Equation (10) is obtained by carrying out a density-weighted average of the non-bonded specie monomer fraction over the bonding and non-bonding regions in the $x-y$ plane at each $z$; hence the dependence of fluid properties on the in-plane coordinates is implicit in equation (10). The bonding and non-bonding regions in any plane are governed by the geometry of the potential model.

Using the free energy thus calculated, the density profile is obtained in the usual manner [36] by the unconstrained minimization of the grand potential.

Equations (10) and (13) are written for the particular case of pure fluid and identical wall sites. Since the TPT1 treats each association site independently, these expressions can be readily generalized for any number of fluid and wall site species simply by independently summing up the contributions due to each type of bonding pair.

It must be emphasized that because the wall sites are assumed to be independent, the theory cannot be expected to do well at high densities of wall sites. If the wall sites are closely distributed, a fluid molecule (or a cluster) bonded with one wall site may block a nearby site, shielding it from other fluid molecules. This amounts to saying that a wall site does affect the bonding at its neighboring sites, which violates the above assumption. Using simulation results for surface coverage and adsorption we find that beyond the wall site density of about $0.75 / \sigma^{2}, \chi^{\mathrm{W}}$ starts decreasing with any further increase in site density, suggesting that all the sites added beyond this point are shielded by fluid molecules already bonded to one of the neighboring wall sites. This effect has also been observed in previous simulation studies by Muller et al. [11]. It is worth mentioning that this limiting value of site density is higher than the surface activation found in most of the real systems such as silica, clay and activated graphite [32]. 


\section{Results and discussion}

We first present the fluid structure and throw some light on the nature of adsorption due to surface association for the system allowing only fluid-wall (F-W) association, followed by a discussion on the interplay between the fluid-wall (F-W) and fluid-fluid (F-F) association.

\subsection{Structure}

The evolution of the fluid density profile with increasing concentration of surface active sites and with increasing association strength (decreasing temperature) is shown in figures 2 and 3 respectively. Figure 2 gives the density distributions of a 4-site associating fluid next to an active wall for surface site densities (a) $0.16 / \sigma^{2}$, (b) $0.25 / \sigma^{2}$, (c) $0.36 / \sigma^{2}$, (d) $0.49 / \sigma^{2}$ at the association strength $\left(\varepsilon^{\mathrm{fw}} / k T\right) 5.0$, while figure 3 corresponds to site density $0.36 / \sigma^{2}$ and association strengths (a) 3.0, (b) 5.0, (c) 7.0 .
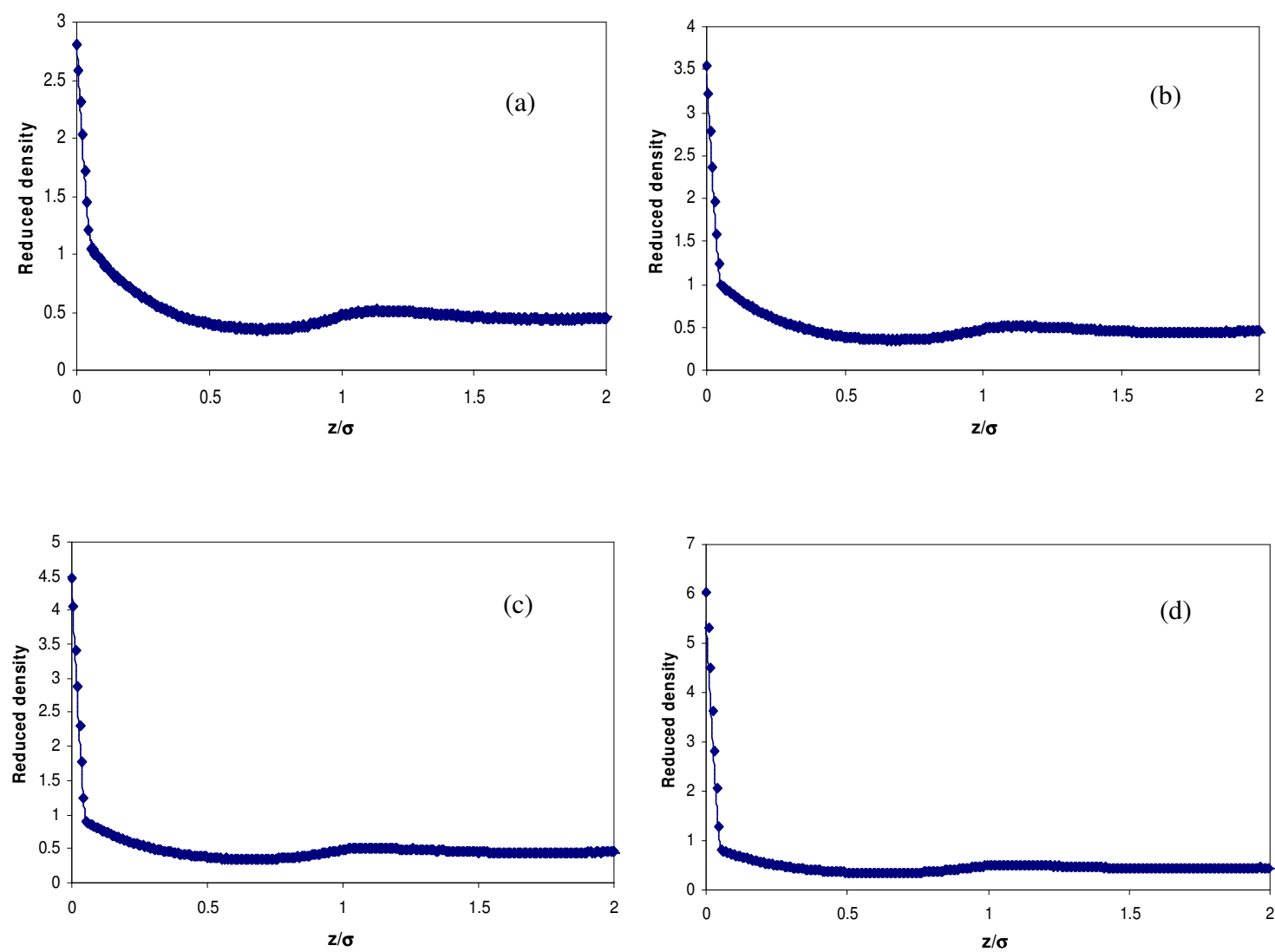

Figure 2. Effect of surface site density. Density profiles of 4-site hard associating fluid at the association strength $\left(\varepsilon^{\mathrm{fw}} / k T\right) 5.0$ and different concentrations of the wall sites. Site densities are (a) $0.16 / \sigma^{2}$, (b) $0.25 / \sigma^{2}$, (c) $0.36 / \sigma^{2}$, (d) $0.49 / \sigma^{2}$. Points are simulations and lines are theory predictions. 

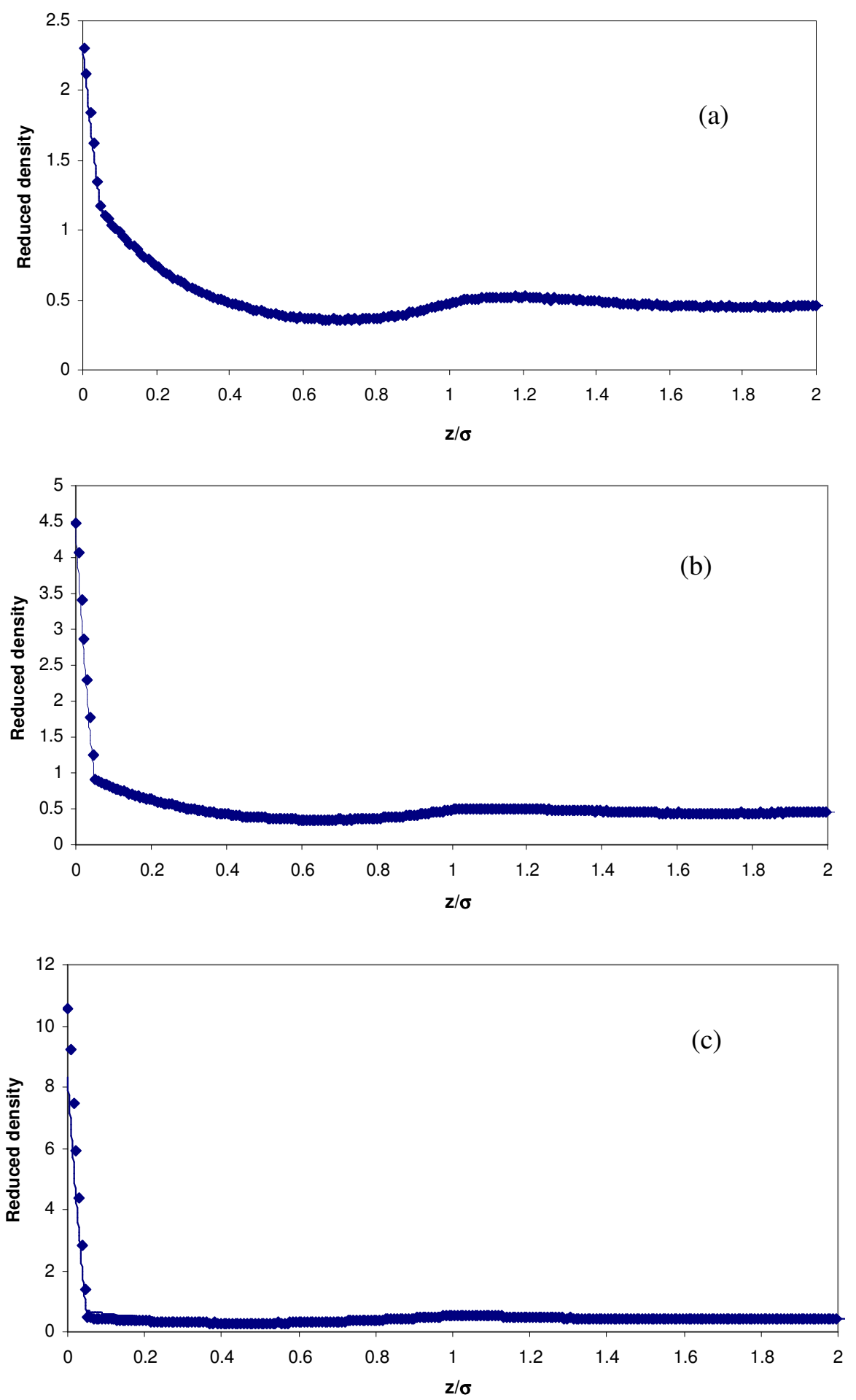

Figure 3. Effect of temperature (association strength). Same as figure 2 for site density $0.36 / \sigma^{2}$ and $\varepsilon^{\mathrm{fw}} / k T$ (a) 3.0 , (b) 5.0, (c) 7.0. 

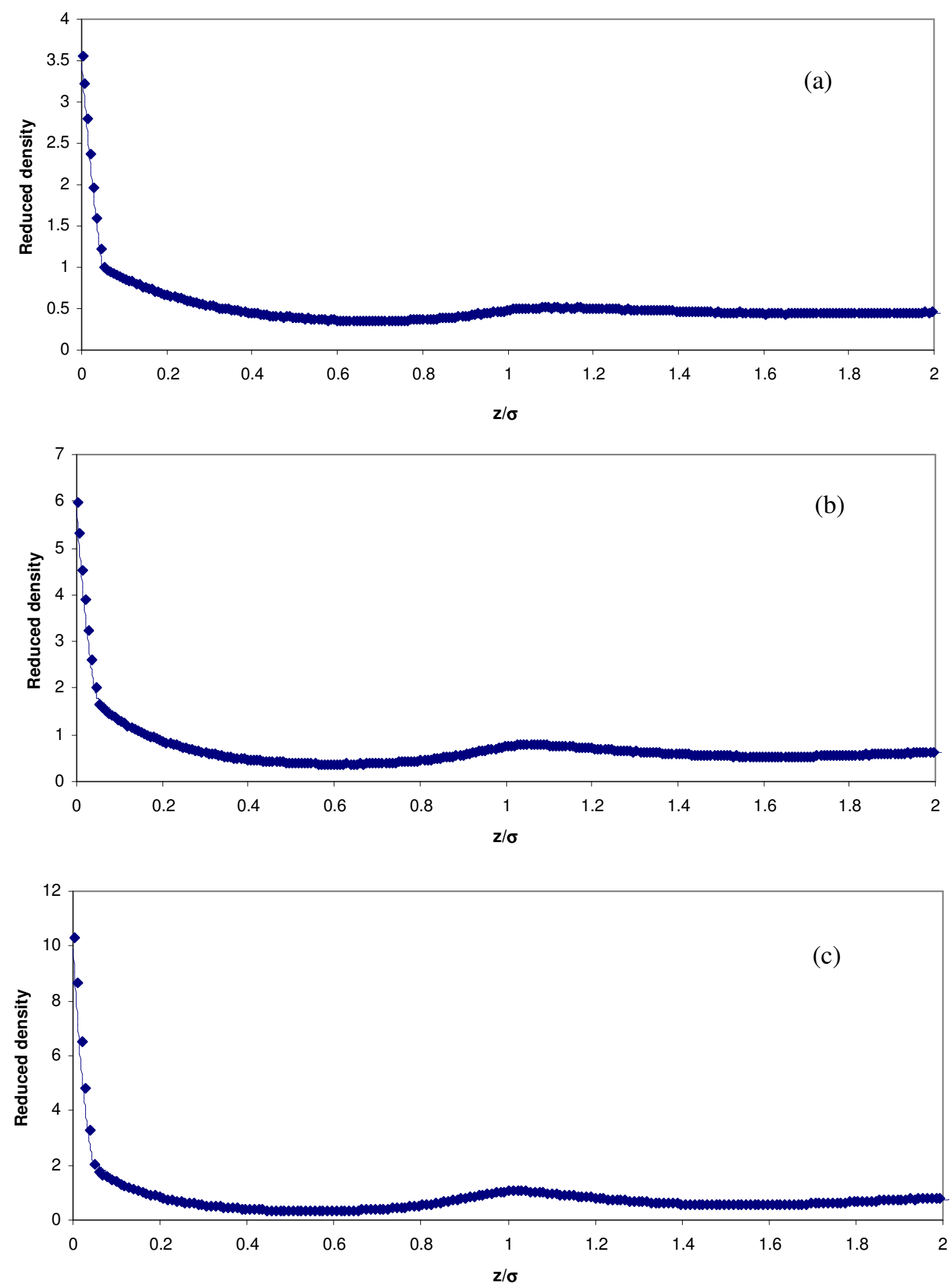

Figure 4. Effect of bulk density. Same as figure 2 for site density $0.36 / \sigma^{2}, \varepsilon^{\mathrm{fw}} / k T$ 5.0 and reduced bulk density $\left(\rho \sigma^{3}\right)$ (a) 0.5 , (b) 0.67, (c) 0.75 . 
The most obvious change in the profiles due to the presence of heterogeneity in the surface is the distinct change in slope of the profile near the bonding cutoff $(0.05 \sigma)$. The profile is roughly linear in the bonding range before following the oscillatory characteristics of a hard-sphere-hard-wall (HSHW) system, thereafter. The density right next to a smooth, non-associating wall, on the other hand, roughly follows a smooth decay. Although the wall bonding volume extends only up to $0.05 \sigma$ from the wall, the density profiles are modified (compared to HSHW system) far beyond the bonding region, extending up to $2 \sigma$. Due to surface association, the positions of the oscillating peaks in the profile are shifted towards the wall, and the fluid density just outside of bonding range but before the first trough is lowered, and that between the first trough and first peak is raised. As surface-association increases (site density increases or temperature decreases), more fluid molecules pile up near the wall. The pronounced volume exclusion effect thus arising is easily seen through the further depressed troughs in the profiles near the wall, outside of bonding range.

The theory accurately predicts all the essential features of the fluid structure all the way up to the wall, even capturing the linear trend in a $0.05 \sigma$ wide bonding range.
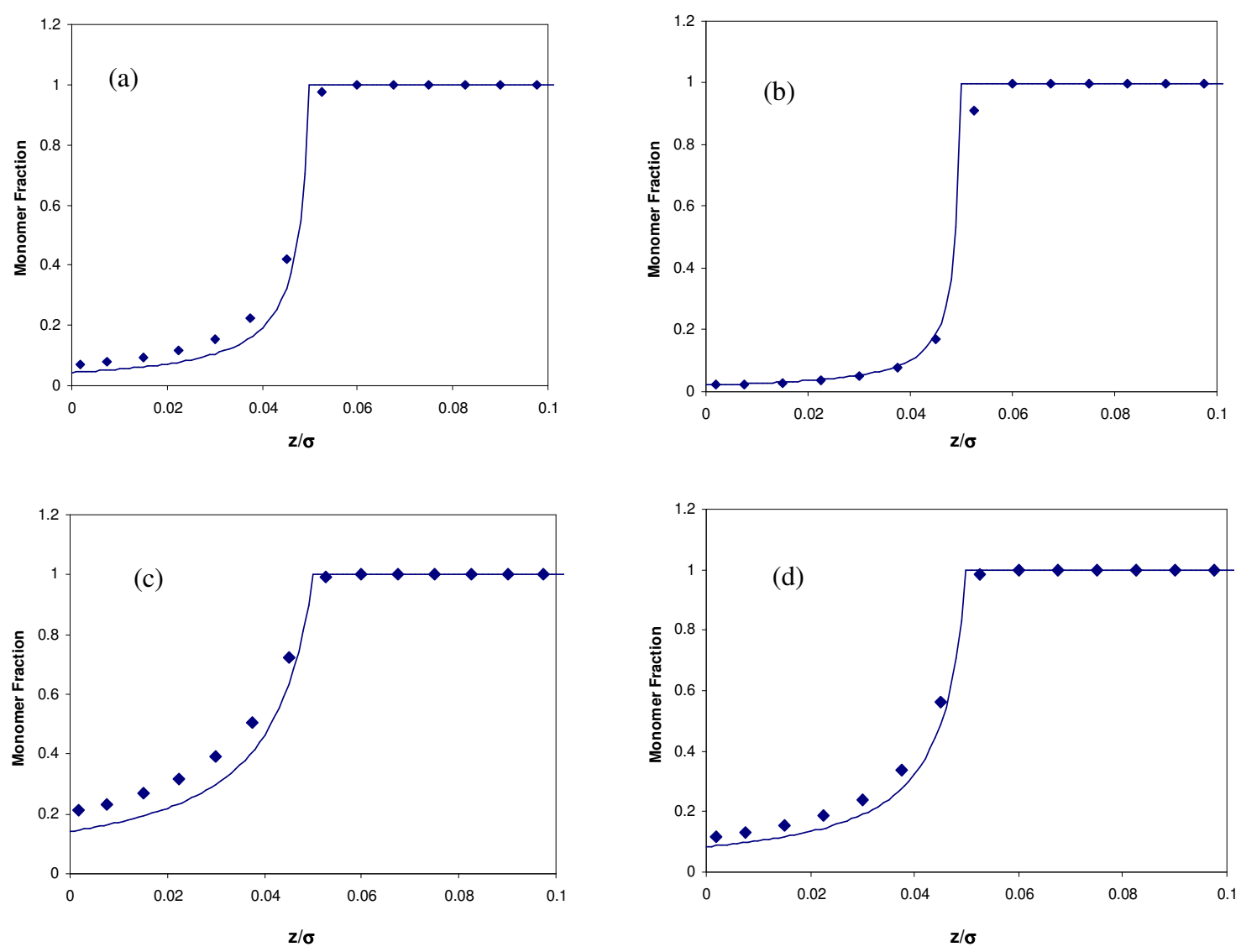

Figure 5. Monomer fraction profiles at different association strengths and site densities. (a) $\varepsilon^{\mathrm{fw}} / k T=7.0$, site density $0.25 / \sigma^{2}$, (b) $\varepsilon^{\mathrm{fw}} / k T=7.0$, site density $0.49 / \sigma^{2}$, (c) $\varepsilon^{\mathrm{fw}} / k T=5.0$, site density $0.36 / \sigma^{2}$, (d) $\varepsilon^{\mathrm{fw}} / k T=5.0$, site density $0.64 / \sigma^{2}$. Points and lines correspond to simulations and theory respectively. 
However, the contact density is slightly underestimated. This may be attributed to one of the simplifying assumptions made in the derivation, discussed in [32]. Further away from the wall, the density profile matches the simulation remarkably well. This should be expected to some extent, since outside the wall bonding range, the fluid is essentially hard sphere, and the theory used here reduces to Tarazona's weighted density functional theory [2] in the absence of association.

Figure 4 shows the density profiles for different chemical potential (or bulk density). The contact density rises and the oscillations in the profile increase with increasing density. The theory again follows the simulations very well, even at high densities.

Figure 5 presents the monomer fraction profiles at different site densities and association strengths. The monomer fraction goes to 1 outside of bonding range, in the absence of any fluid-fluid association. Although the density profile was linear, the monomer fraction profile has a distinct curvature, which is due in part, to the geometry of the association potential. It is remarkable that the theory captures this curvature even over such a small range and at high association strengths, with appreciable accuracy.

\subsection{Adsorption / surface coverage}

An interesting feature of the system, elucidated by the theory, and confirmed by simulations, is that surface association site coverage in the absence of fluid-fluid bonding proceeds through monolayer formations. This however, is not fortuitous. As a molecule is attached to a surface site, no lateral interaction between the neighbors is allowed and the surface sites can have 0 or 1 occupancy. Moreover, the attractive forces of the wall decay rapidly with distance, and only the molecules near the wall

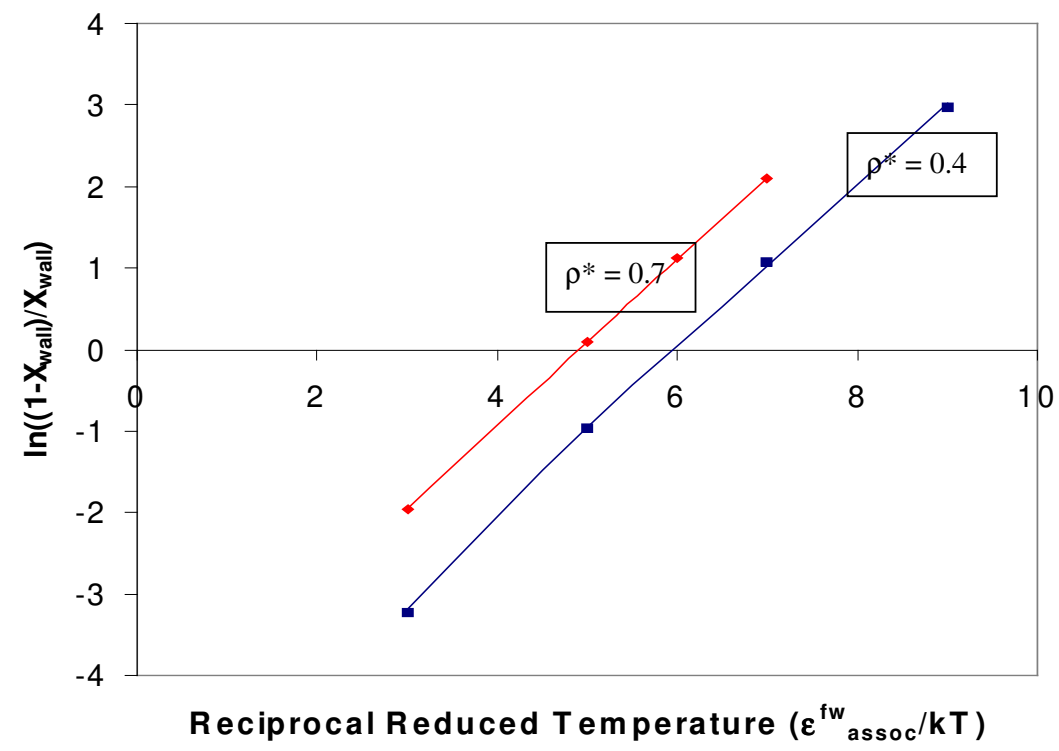

Figure 6. Monolayer adsorption characteristics of a 4-site fluid associating only with surface sites. Points and lines are simulations and theory respectively. 
are affected. These features of our system are common to Langmuir's monolayer adsorption model, where the wall-fluid association is short ranged (extending up to $5 \%$ of molecular diameter), steric effects are ignored, and only one fluid molecule can bond to any given wall site. The linear plots of $\ln \left[\left(1-\chi^{\mathrm{W}}\right) / \chi^{\mathrm{W}}\right]$ versus inverse reduced temperature in figure 6 clearly bring out the monolayer adsorption characteristics of the system. The excellent agreement between the theory and the simulations in figure 6 is noteworthy.

\subsection{Introducing fluid-fluid association}

With the introduction of fluid-fluid bonding in the system, the equilibrium distribution of fluid molecules is determined by the competing energetic and entropic effects. The clusters formed as a result of fluid-fluid association tend to stay away from the wall to avoid entropic penalty. On the other hand, surface-fluid association tends to draw the fluid molecules near the surface by lowering the system energy.

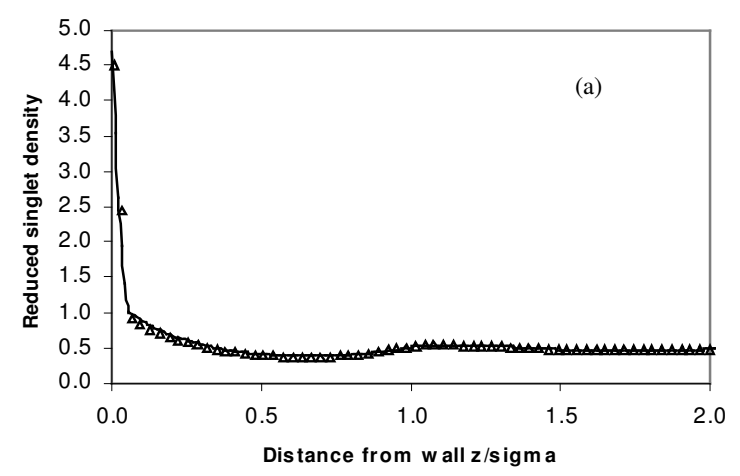
Figure 7 shows that the theory accurately predicts the fluid structure for different fluid-fluid and fluid-wall association strengths for water-like fluids (4 association sites). The fluid structure in this case is also changed significantly due to surface adsorption. Keeping the fluid-fluid association constant, increasing the fluid-wall association leads to a higher density at contact, as expected, but an interesting behavior is observed upon increasing the F-F association strength at constant F-W association strength. Table 1 gives the fractional wall coverage for different values

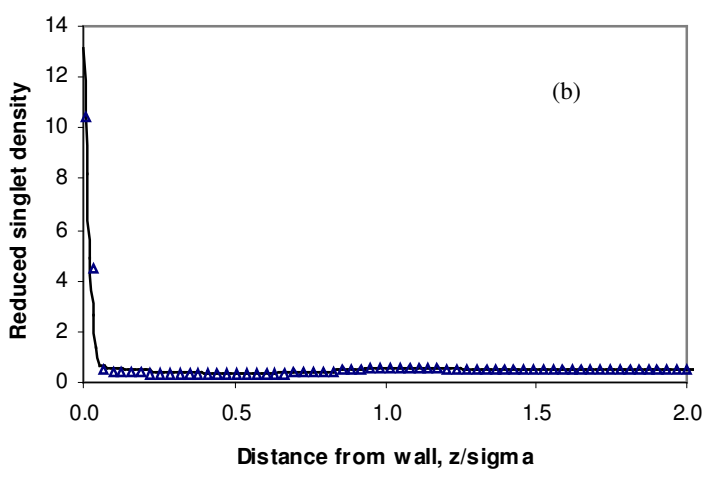

Figure 7. Same as figure 2, with both F-W and F-F associations active (a) $\varepsilon^{\mathrm{ff}} / k T=5.0, \varepsilon^{\mathrm{fw}} / k T=3.0$, (b) $\varepsilon^{\mathrm{ff}} / k T=$ $3.0, \varepsilon^{\mathrm{fw}} / k T=5.0$. of F-F and F-W strengths at different bulk densities. The theory (in excellent agreement with simulations) suggests that the F-W association remains unaffected by the F-F association at all bulk densities.

The reason for this seemingly strange behavior lies in the particular geometry of the 4-site fluid. Because of the tetrahedral symmetry of the arrangement of the sites on the surface, for any orientation of such a molecule next to the wall, one of the sites will always be facing away from and hence cannot bond to any of the remaining fluid molecules no matter how strong the F-F association strength is. This site is however, available to bond with a surface site, hence the surface coverage is only determined by 
Table 1. Fractional wall coverage from theory and simulations for different combinations of F-F and F-W association strengths at different bulk densities.

\begin{tabular}{|c|cccc|}
\hline Bulk Density & $\varepsilon^{\mathrm{fw}} / k T$ & $\varepsilon^{\mathrm{ff}} / k T$ & $X_{\text {wall }}(\mathrm{DFT})$ & $X_{\text {wall }}(\operatorname{sim})$ \\
\hline 0.7 & 7 & 3 & 0.1107 & 0.112 \\
& 7 & 5 & 0.1113 & 0.1104 \\
& 7 & 7 & 0.1105 & 0.1108 \\
0.7 & 5 & 3 & 0.4806 & 0.4861 \\
& 5 & 5 & 0.4805 & 0.4902 \\
0.5 & 5 & 3 & 0.7205 & 0.7236 \\
& 5 & 5 & 0.7162 & 0.728 \\
0.7 & & & & \\
& 3 & 3 & 0.8772 & 0.8798 \\
& 3 & 5 & 0.8782 & 0.8771 \\
\hline
\end{tabular}

the strength of the F-W association. To test this reasoning we performed simulations and theory calculations for 1-site fluids that do not possess the above mentioned feature of tetrahedral symmetry and found that the surface coverage was indeed

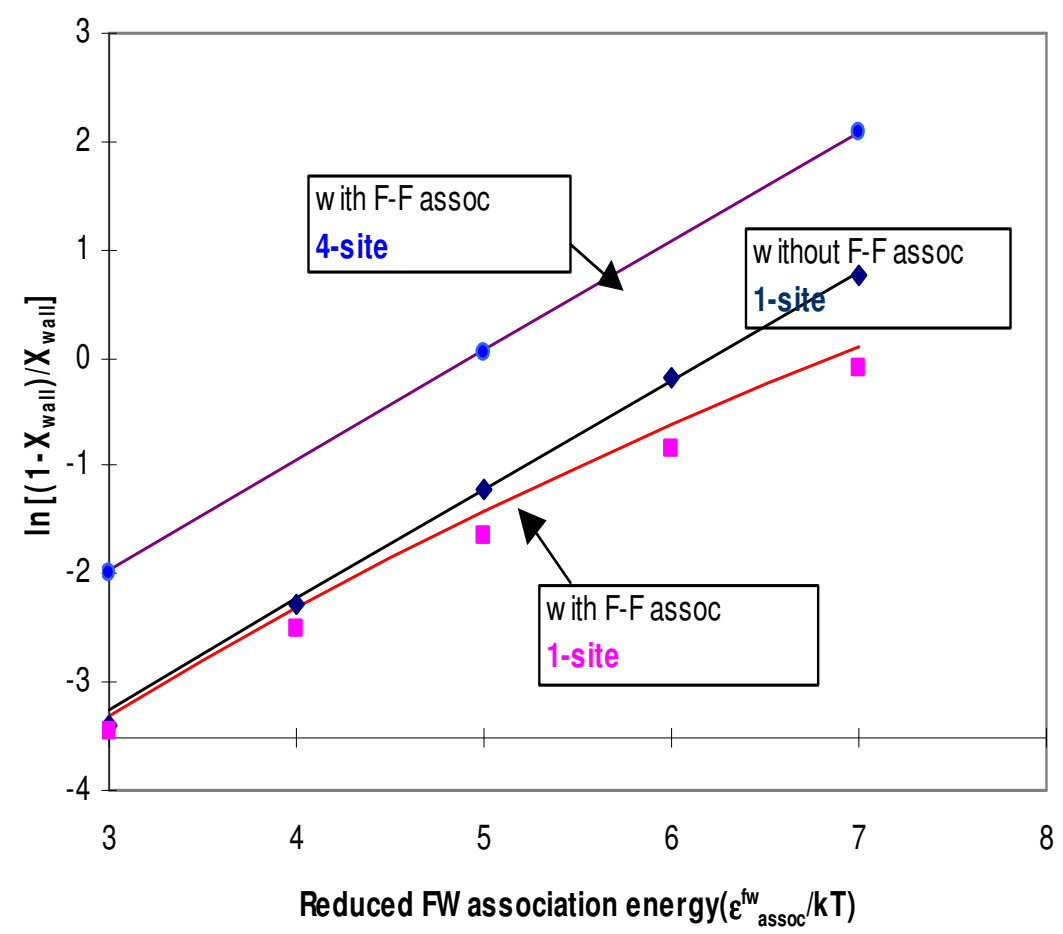

Figure 8. Fluid-fluid association changes the monolayer adsorption characteristics of a 1-site fluid, but that of a 4-site fluid remains unchanged because of the particular arrangement of surface sites. 
affected by F-F association. Figure 8 shows surface coverage plots, similar to figure 6 highlighting the effect of introducing F-F association in 4 and 1 site fluid systems. Since the surface coverage in a 4-site fluid was independent of F-F association, the adsorption is through monolayer formations, as in the case without F-F association. While in a 1-site fluid, the adsorption deviates from monolayer behavior because of F-F association. The theory is once again in excellent agreement with simulations for each case.

\section{Conclusions}

We have presented a perturbation DF theory based on TPT1, to describe adsorption of associating fluids near surfaces with discrete heterogeneity, introduced by the presence of active sites across the surface. The most important feature of the theory is that it accounts for the dependence of fluid properties on the in-plane coordinates without actually solving a full 3D DFT. It employs the geometry of the potential model to simplify the 3D functionals to their 1D forms. The theory correctly predicts the total, bonded and non-bonded specie distribution away from the wall, adsorption and wall coverage in these systems with $3 \mathrm{D}$ inhomogeneity, at the computational expense of a 1D DFT; over a range of bulk fluid density, surface site density and temperature. Since the theory captures the monolayer adsorption characteristics of associating fluids due to surface association with remarkable accuracy, it can also be seen as an extension of Langmuir adsorption isotherm (accurate only for ideal gases) to hard associating fluids, at all fluid densities.

\section{Acknowledgements}

We gratefully acknowledge the Robert A. Welch Foundation for the financial support of this work.

\section{References}

1. Chmiel C., Karykowski K., Patrykeijew A., Rzysko W., Sokolowski S. // Molec. Phys., 1994, vol. 81, p. 691.

2. Tarazona P., Bettelo M.M., Evans R. // Molec. Phys., 1987, vol. 60, p. 573.

3. Vanderlick T.K., Scriven L.E., Davis H.T. // J. Chem. Phys., 1989, vol. 90, p. 2472.

4. Rocken P., Tarazona P. // J. Chem. Phys., 1996, vol. 105, p. 2034.

5. Rocken P., Somoza A., Tarazona P., Findenegg G.H. // J. Chem. Phys., 1998, vol. 108, p. 8689.

6. Huerta A., Pizio O., Bryk P., Sokolowski S. // Molec. Phys., 2000, vol. 98, p. 1859.

7. Zygmunt J.R., Pizio O., Rzysko W., Sokolowski S., Sokolowska Z. // J. Colloid. Interf. Sci., 2001, vol. 241, p. 169.

8. Segura C.J., Chapman W.G. // Molec. Phys., 1995, vol. 86, p. 415.

9. Segura C.J. Ph.D. Dissertation. Rice University, 1997.

10. Segura C.J., Chapman W.G., Shukla K.S. // Molec. Phys., 1997, vol. 90, p. 759. 
11. Muller E.A., Gubbins K.E. // Carbon, 1998, vol. 36, p. 1499.

12. Muller E.A., Gubbins K.E. // Carbon, 1998, vol. 36, p. 1433.

13. Muller E.A., Rull L.F., Vega L.F., Gubbins K.E. // J. Phys. Chem., 1996, vol. 100, p. 1189.

14. Muller E.A., Vega L.F., Rull L.F., Gubbins K.E. // Molec. Phys., 1995, vol. 85, p. 9.

15. McCallum C.L., Bandosz T.J., McGrother S.C., Muller E.A., Gubbins K.E. // Langmuir, 1999, vol. 15, p. 533.

16. Lee S.H., Rossky P.J. // J. Chem. Phys., 1994, vol. 100, p. 3334.

17. Kovalenko A., Pizio O., Henderson D., Sokolowski S. // J. Colloid Interf. Sci., 1996, vol. 182 , p. 407.

18. Vakarin E., Duda Y., Holovko M. // J. Chem. Phys., 1997, vol. 107, p. 5569.

19. Delville A., Sokolowski S. // J. Phys. Chem., 1993, vol. 97, p. 6261.

20. Bridgeman C.H., Skipper N.T. // J. Phys.: Condens. Matter, 1997, vol. 9, p. 4081.

21. Shroll R.M., Smith D.E. // J. Chem. Phys., 1999, vol. 111, p. 9025.

22. Tunega D., Haberhauer G., Gerzabek M.H., Lischka H. // Langmuir, 2002, vol. 18, p. 139.

23. Deville A. // Langmuir, 1992, vol. 8, p. 1796.

24. Chatterjee A., Iwasaki T., Ebina T., Hayashi H. // Appl. Surf. Sci., 1997, vol. 121/122, p. 167.

25. Wertheim M.S. // J. Statist. Phys., 1984, vol. 35, p. 19.

26. Wertheim M.S. // J. Statist. Phys., 1984, vol. 35, p. 35.

27. Wertheim M.S. // J. Statist. Phys., 1986, vol. 42, p. 477.

28. Wertheim M.S. // J. Statist. Phys., 1986, vol. 42, p. 459.

29. Tripathi S., Chapman W.G. // J. Chem. Phys., 2003, vol. 118, p. 7993.

30. Meister T.F., Kroll D.M. // Phys. Rev. A, 1985, vol. 31, p. 4055.

31. Rosenfeld Y. // Phys. Rev. Lett., 1989, vol. 63, p. 980.

32. Tripathi S., Chapman W.G. // J. Chem. Phys. (submitted).

33. Tripathi S., Chapman W.G. // J. Chem. Phys. (in preparation).

34. Frink L.J.D., Sallinger A.G. // J. Comput. Physics, 2000, vol. 159, p. 407.

35. Frink L.J.D., Sallinger A.G. // J. Comput. Physics, 2000, vol. 159, p. 425.

36. Evans R. Fundamentals of Inhomogeneous Fluids. New York, Marcel-Dekker, 1992. 


\title{
Адсорбція асоціативних флюїдів на активних поверхнях. Теорія функціоналу густини
}

\author{
С.Тріпаті, У.Г.Чепмен \\ Відділення хімічної інженерії, Університет Райса, \\ Гюстон, TX 77005, США
}

Отримано 7 травня 2003 р., в остаточному вигляді - 4 серпня 2003 p.

Ми застосовуємо теорію функціоналу густини (ТФГ) до опису систем, у яких молекули асоціативних плинів можуть зв'язуватися (або асоціювати) з дискретними локалізованими функціональними групами, приєднаними до поверхонь, на додаток до інших молекул флюїду. Для таких систем як вода, що адсорбується на активованому вуглеці, кремнії, глині, це є реалістичнішою моделлю для врахування поверхневої неоднорідності, ніж використання неперервного липкого потенціалу поверхня-плин, що застосовується в більшості теоретичних робіт з адсорбції на неоднорідних поверхнях. Асоціація моделюється в межах першого порядку термодинамічної теорії збурень (ТТЗ1). Нова теорія дає точне передбачення розподілу зв'язаних і незв'язаних зразків і адсорбційної поведінки при різних значеннях об'ємного тиску і асоціативної взаємодії поверхня-плин і плинплин. Проведено порівняльний аналіз між асоціативною взаємодією поверхня-плин і плин-плин для флюїдів зі складними асоціативними вузлами і обговорено їх вплив на адсорбцію. Теорія, підкріплена симуляціями, показує, що міра і природа адсорбції змінюється зі зміною кількості вузлів асоціації на молекулах флюїду.

Ключові слова: адсорбція, асоціативні флюїди, активні поверхні, теорія функціоналу густини

PACS: 68.35.Dv, 68.43.-h, 68.43.De, 68.43.Fg, 71.15.Mb 